\title{
Quality of service and reliability in optical network
}

\author{
Jitendra Saxena ${ }^{1}$ and Aditya Goel ${ }^{2}$ \\ ${ }^{1}$ Department of Information Technology \\ Maulana Azad National Institute of Technology, Bhopal, India \\ email-jsaxena2007@rediffmail.com \\ ${ }^{2}$ Department of Electronics \& Communication Engineering \\ Maulana Azad National Institute of Technology, Bhopal, India \\ Email-adityagoel2@rediffmail.com
}

\begin{abstract}
Eminence of service is a extremely significant and critical concern for maintaining optical network with respect signal degradation. Queuing theory is an vital parameter to maintain voice video and data eminence of service. The network consists of different type of eminence of service model with priority of service with special reference to delay, speed and jitter.
\end{abstract}

\section{KEYWORDS}

Priority of service, Video on demand, IP packet delay queuing, packet queuing.

\section{INTRODUCTION}

Reliability has been well standard as an imperative design parameter in the plan of modern high-momentum networks[1,2]. While past approaches offer either $100 \%$ protection or partial protection referring to path protection span protection or link protection, connections in real networks may have multiple eminence of service requirements, reliability and availability requirements. The modern issue in eminence of service will lead to modern design including Pre design and post design phase towards integrated and differentiation solution and approach that will support multi provisioning platform like voice video and data.

Evidence of this trend over the last years is the introduction of concepts such as quality of service (QoS) [1, 2], and differentiated services [3, 4] that provide multiple levels of service performance in the same network [5, 6].The benefit of optical fiber deployment is low loss span with high band width capacity for the subscribers. In addition to the cost of network downtime, there is also the cost of service degradation like network slowness, latency and jitter are far common than network downtime. They also affect more users and last longer because they are more difficult to diagnose. Simultaneously increase in competitiveness among operators has led to an increased focus on quality of service (QoS). The assessment of reliability and quality of service is therefore the basis for the trade-off between availability cost and performance.

\section{Methodology}

There are many schemes to enhance return on investment and to maximize profit with reduced network cost. Distinct degree of service include high priority of service low priority service and medium priority of service and these distinct are manage efficiently by scheduling algorithm and service level agreement . Data rate is increasing exponentially and to accommodate demand and flexible bandwidth different types of queuing theory and pre configuration algorithm are designed for high eminence of service like video on demand and high tech multi media application. Cooperate giants requires service level agreement guarantee from different service 
providers. The network is compared of web client/server and a video client/client model. A sub network is a token ring network which represent bottleneck. The first level of Queuing schemes is for individual, second level of queuing is for distinct degree of customer and third level is for different channels metro network for channels 1,2,3. All types of traffic are classified as type of service. Video traffic is queued and prioritized in the firewall by using WFQ. However video traffic and delay is dependent on http traffic.

First level scheduler to ensure QoS for Individual

1. job significant services

2. organization connectivity

3. Video consultation

4. Internet transfer

- Second level scheduler to ensure QoS For customer

1.High Priority client

2.Medium Priority client

3.Low Priority client

- Third level scheduler to ensure QoS for tunnels

1. Metro network channel 1

2. Metro network channel 2

3. Metro network channel 3

\section{RESULT AND DISCUSSION}

Routers support multiple queues for each type of service. Queue 4 receives type of service 4 traffic; queue 3 receives type of service 3 traffic as shown in Fig 3.1. Queues are serviced using "Weighted Fair Queuing can be enabled on each interface in "advanced routers. Queuing profiles and queuing processing mechanism are set in attribute "QoS information scenario in " IP address information" compound attribute. Queuing profiles define the number of queues and the classific tion scheme. Queuing profile are defined in the QoS configuration. In this design the IP Packet Queuing mechanism differentiates traffic between queues based on the type of service(TOS). Queues send traffic proportionally to their weight. In this design queues with high index have higher weight. As a result of this classification traffic with higher type of service gets better delay. Queue 3 and 4 get their share but let other queues starving of bandwidth. The only difference in weighted Fair Queuing and weighted Fair Queueing with low latency queue(LLQ) is in the IP Packet Queuing profile detail setting where queue 1 is configured to be a low latency Queue(LLQ). The LLQ is a strict priority queue functioning within the regular weighted Fair Queuing scheduling environment. It receives absolute precedence over the other queues which mean that no other queue in the system can be serviced unless the LLQ is empty. If the LLQ is empty, other queues are serviced according to the regular "weighted Fair queuing mechanism. Traffic is queued in router A because of the bottleneck. Queue 1 which is configured to be LLQ get the highest priority with highest degree of reliability and thus the highest share of the bandwidth and lowest end to end delay.Queue 4 which has the highest weight among the other queues gets afull share of the bandwidth. Queues 2 and 3 get starved and have higher delays as shown in Fig 3.2 . 
International Journal of Distributed and Parallel Systems (IJDPS) Vol.3, No.1, January 2012

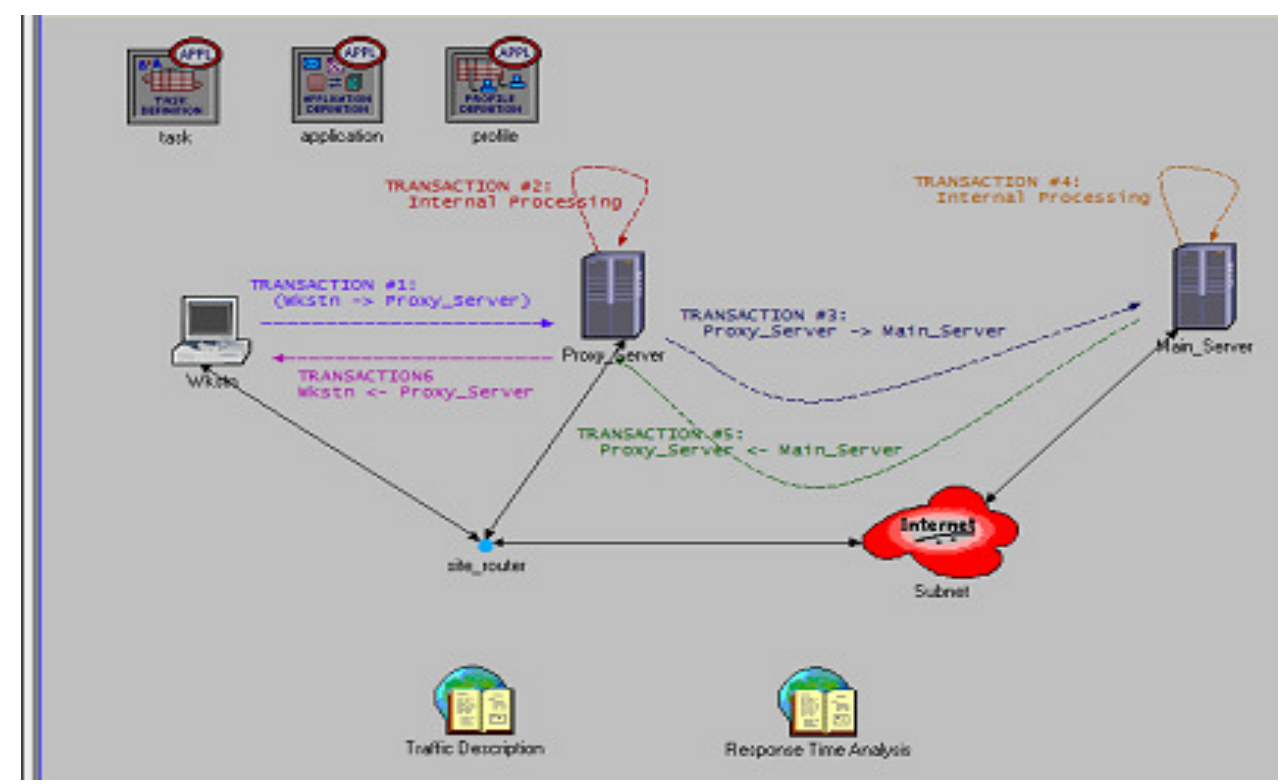

Fig3.1 Design Layout for Hierarchical Quality of Service.

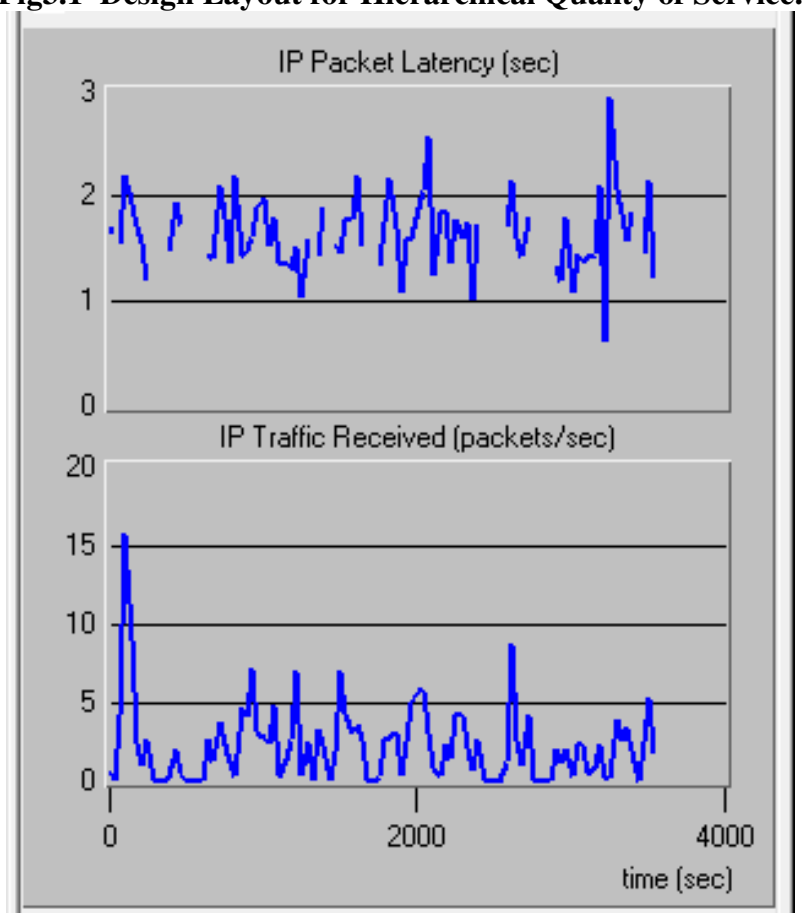

Fig.3.2 Result for IP Packet Queuing, between Events per second \& Time 
International Journal of Distributed and Parallel Systems (IJDPS) Vol.3, No.1, January 2012

\section{CONCLUSION}

We investigate various weighted fair queuing with hierarchical Quality of service model and approach with OPNET simulation tool. The result shows in terms of fairness and delay performance. The losses of packets from higher priority video traffic can be eliminated using IP Packet Queuing with LLQ.

\section{References}

[1] M.J. Fischer and D.M.B. Masi, "Modeling Overloaded Voice over Internet Protocol Systems," Telecomm. Rev., 2006;

[2] http://www.noblis.org/TelecommunicationsReview.html.

[3] Zirong Guo et.al " Simulation and analysis of weighted Fair Queuing algorithm in OPNET" International conference on computer modelling and simulation IEEE 2009

[4] http://www.noblis.org/BusinessAreas/ProgramManagement Acquisition/2591_final_ncs_ngps_Modeling_and Simulation_Group_MFischer_DMasi_10.03.2006. pdf.

[5] http://www.noblis. org/BusinessAreas/ProgramManagementAcquisition/ATS05_final4 Spring_2005.pdf.

[6] www.opnet.com

[7]www.noblis.org/BusinessAreas/ProgramManagementAcquisition/ICTSM_2005VoIP_Performance_M odels_2005--_final3.pdf.

[8] D. Gross and C. M. Harris, Fundamentals of Queueing Theory, 3rd ed., John Wiley, 1998.

[9] Cisco IOS Quality of Service Solutions Configuration Guide, Release 12.2, Part 2:

CongestionManagement;

http://www.cisco.com/univercd/cc/td/doc/product/software/ios122/122cgcr/fqos_c/.Additional

Resources on Queuing Disciplines

Jitendra Saxena is is research scholar from MANIT Bhopal and pursuing his Ph.D in Diff Reliability \& Availability, QoS in WDM optical network, working with optical layer protection and restoration. Linking IP over WDM layer by means of virtual server.

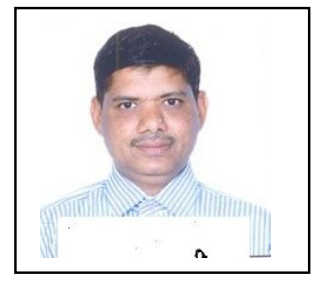

